\section{Das Auslaufmodell}

\author{
E. Taverna
}

Der diesjährige Carl-Bertelsmann-Preis für «exemplarische Lösungen auf dem Gebiet der medizinischen Versorgung" ging an die Schweiz für das neue Krankenversicherungsgesetz KVG und an Holland für das dort etablierte System der Haus- und Familienärzte.

Ist das Schweizerische Kollegium für Hausarztmedizin KHM ebenfalls preisverdächtig?

Als Dachorganisation der Gesellschaften für Allgemeinmedizin SGAM, Innere Medizin SGIM und Pädiatrie SGP wurde es, zusammen mit den fünf medizinischen Fakultäten und der Schweizerischen Akademie der Medizinischen Wissenschaften SAMW, 1994 gegründet. Präsident des Stiftungsrates seit Januar 1999 ist Dr. med. Werner Bauer, Internist in Küsnacht. Die drei Fachgesellschaften, die sich als "Grundversorger" bezeichnen, erhalten von der SAMW einen jährlichen Beitrag von 50000 Franken, mindestens bis 2003. Die Akademie hat ein Interesse daran, das Potential der Praxisforschung mit der aktuellen akademischen Forschung zusammenzubringen. Der Jahresbericht des KHM von 1999 nennt als Hauptziele den Ausbau der Forschung und das Bearbeiten gemeinsamer Probleme der Trägergesellschaften. Arbeitsgruppen befassen sich mit der Aus-, Weiter- und Fortbildung, Praxisassistenz, Praxislabor und Qualitätssicherung. Für die noch wenig entwickelte Forschung soll ein Netz interessierter Teilnehmer aufgebaut werden.

\section{Vom Phantombild ...}

Ein Grundsatzpapier der Schweizerischen Medizinischen Interfakultätskommission SMIFK sprach 1982 von einer "gewissen Fehlentwicklung", weil die neue Medizinalprüfungsverordnung die Allgemeinmedizin vernachlässigt hatte. Zur Frage, wer für die allgemeinmedizinische Versorgung optimal gerüstet sei, ob Spezialist oder Generalist, Hausarzt oder Familienmediziner, gab es keine gemeinsame Meinung. Einig waren sich die Experten einzig über die Feststellung, dass während des Studiums die Berufsmotivation zum "Allgemeinpraktiker» abnimmt. Die Ursachen wurden in der Vorbildfunktion der ausnahmslos spezialisierten, klinischen Lehrer, im Mangel an Information und in fehlenden, geeigneten Rotationsstellen geortet. Einig war man sich auch darin, den Begriff "Allgemeinmedizin" möglichst breit als "Hausarztmedizin" oder als "ambulante Medizin" zu interpretieren.
Die SGAM formulierte 1991 nach britischem Vorbild einen Katalog über die "Merkmale eines guten Hausarztes». Der Abschnitt "Klinische Kompetenzen» erwähnt keine fachspezifischen Inhalte, die Forderungen treffen auf jeden behandelnden Arzt zu. Die polyvalente Fachkompetenz ist kein Thema. Auch die Europäische Union der Allgemeinpraktiker UEMO formulierte 1995 Berufsziele, die keine Fachrichtung beinhalten. Liest man, was der Vorstand zum «New millennium of outstanding ideas" zu sagen hat, dann bleiben dem Allrounder und Generalisten die mit Spezialisten unterversorgten, ländlichen Regionen und die ökonomisch wenig attraktiven Gesellschaftsschichten.

20 Jahre nach der diagnostizierten «Fehlentwicklung» und 6 Jahre nach Gründung des KHM sind die vorgeschriebenen Lehrinhalte zum FMH Allgemeinmedizin obsolet geworden. Das Modell ist am Auslaufen.

\section{... zur helvetischen Zukunft}

Wenn laut Bauer Tessiner Kollegen über die Verschmelzung von SGIM und SGAM nachdenken, ist das konsequent und wegweisend. Denn auch der Allgemeininternist ist ohne seine Subspezialitäten mit ähnlichen Problemen wie der Allgemeinarzt konfrontiert. Was in einer Fakultät nicht einen ordentlichen Lehrstuhl hat, in der Forschung nicht mithält und kein eigenständiges Prüfungsfach ist, existiert dort nicht. Einzig Bern hat fünf Praktikern einen professoralen Sitz eingeräumt. Die anderen Fakultäten haben, auf Druck von aussen, einer «Fakultären Instanz für Hausarztmedizin» eine Nische eingeräumt. Immerhin. Was dort geleistet wird, ist beachtlich und verdient Anerkennung. Doch ein Patchworkberuf, der alle seine Fächer den "Etablierten» abbettelt, bleibt dem Goodwill der Dekane und Chefärzte ausgeliefert. Und die sind unter Druck und schauen erst einmal für sich. Die Spitäler könnten zudem mit der Streichung von Ausbildungsstellen Geld sparen und ihre Spezialisten gewinnbringend in der Ambulanz beschäftigen. Die Kunden murren zwar über die Prämien, doch sie holen sich Rat in der Leistungsshow von Leutschenbach und die Versicherer jonglieren pausenlos mit neuen Modellen und haben die Lust an Hausarztsystemen verloren. Qualität wird mit Spitzenmedizin gleichgesetzt, und wer politisch das Sagen hat, ist privat versichert.

Jeder Bereich des Gesundheitswesens ist bis zur Basis durchspezialisiert und in Zukunft mit tarifwirksamen Fähigkeitszeugnissen abgedichtet. Das Kollegium für Hausarztmedizin ist heute am Anfang. Gefragt sind neue, mutige Ideen, die aus den überholten Trägergesellschaften einen neuen Facharzt hervorgehen lassen. An Hinweisen fehlt es nicht, denn wer jetzt mit dem Studium beginnt, ist mehrheitlich weiblich, teilzeitorientiert und teamfähig. Der einsame Revierkämpfer ist tot, es lebe die neue Grundversorgerin! 